\title{
Cognition and the supplementary motor complex
}

\author{
Parashkev Nachev, Christopher Kennard and Masud Husain
}

In our recent Review article (Functional role of the supplementary and pre-supplementary motor areas. Nature Rev. Neurosci. 9, 856-869 (2008) $)^{1}$, we discussed the role of the supplementary motor complex (SMC) in different aspects of action. Leek and Johnston (Nature Rev. Neurosci. 19 Dec 2008 (doi:10.1038/nrn2478-c1)) ${ }^{2}$ draw attention to an interesting matter that we did not discuss: the relation between SMC activity and the performance of tasks seemingly remote from action. The example they refer to is that of mental rotation, but several other activities might just as easily be considered. Our omission of these is not accidental, for the following reasons.

First, it is much more difficult to divorce a putative cognitive process from action than is commonly supposed. Indeed, nothing could be said about a mental activity that was not anchored at some point in the subject's behaviour, even if only in a verbal report (which is, of course, a kind of action). Attempts to dissociate non-action components on the basis of timing (for example, by introducing a delay between stimulus and response, as in the classic delayed-saccade task) are fundamentally insecure because as long as neural activity has temporal priority over an action it may be materially contributing to it. Nor is it possible to rely on the omission of action in a particular instance, because the mere potentiality of action is sufficient to explain activation in such situations: the brain, as we know, is a predictive system ${ }^{3}$. Moreover, omissions are actions themselves, legally and semantically ${ }^{4,5}$, and resemble commissions behaviourally and neurally ${ }^{6}$. Thus, when two sets of circumstances differ in their habitual association with some potential action, their contrast will always be explicable by actionrelated activity regardless of whether an action is performed or not. In short, actions are characterized by their conditions as much as by their means or ends.

Second, although when we speak of actions we often have limb movements in mind, speech and visual exploration are also forms of action. As there can be no abstraction without symbolism, and no symbolism without a market of symbolic exchange, such as language, it is generally impossible to eliminate incidental language-related activation in circumstances of any kind of abstract cognition. Equally, SMC activity related to eye movements will always contaminate spatial tasks such as mental 'rotation' of figures. Instructing the subject to maintain fixation would merely make activity in the SMC explicable by the unnatural withholding of gaze shifts ${ }^{7}$. Thus, a task construed to be purely cognitive, such as mental rotation, might nevertheless be associated with action-related neural activity.

Third, that a behaviour and a mental activity may be analogously conceptualized is, of course, no evidence that they share a neural substrate. Spatial rotations in the mind are not rotations in an inner space ${ }^{8}$. Moreover, there is no evidence that vector transformation for abstract processes or motor control is indeed a common currency for the SMC. Nevertheless, we are grateful to Leek and Johnston for raising these issues. This entire area of brain research remains a very difficult one and it is crucial, if the enterprise is to succeed, that we remain vigilant about potential conceptual problems associated with interpretations of existing and future - experimental studies.

1. Nachev, P., Kennard, C. \& Husain, M. Functional role of the supplementary motor and pre-supplementary motor areas. Nature Rev. Neurosci. 9, 856-869 (2008).

2. Leek, E. C. \& Johnston, S. J. Functional specialization in the supplementary motor complex. Nature Rev. Neurosci. 19 Dec 2008 (doi:10.1038/nrn2478-c1).

3. Koerding, K. P. \& Wolpert, D. M. Bayesian integration in sensorimotor learning. Nature 427, 244-247 (2004).

White, A. R. Grounds of Liability (Oxford Univ. Press, New York, 1985).

5. Hacker, P. M. S. Human Nature: the Categorial Framework (Blackwell, 2007).

6. Hanes, D. P. \& Schall, J. D. Countermanding saccades in macaque. Vis. Neurosci. 12, 929-937 (1995).

7. Curtis, C. E., Cole, M. W., Rao, V. Y. \& D’Esposito, M. Canceling planned action: an fMRI Study of countermanding saccades. Cereb. Cortex 15 , 1281-1289 (2005).

8. Bennett, M. R. \& Hacker, P. M. S. Philosophical Foundations of Neuroscience (Blackwell, Oxford, Victoria, 2003).

Parashkev Nachev and Masud Husain are at the Institute of Cognitive Neuroscience and Institute of Neurology, University College London, Alexandra House, 17 Queen Square, London, WC1N 3AR, UK.

Christopher Kennard is at the Department of Clinical Neuroscience, Imperial College London, St Dunstan's Road, London, W6 8RP, UK.

Correspondence to M.H. e-mail:m.husain@ion.ucl.ac.uk 